10 - ORIGINAL ARTICLE

TECHNICAL SKILL

\title{
Experience report on teaching surgical technique without animal use ${ }^{1}$
}

\author{
Gustavo Maltez de Andrade \\ Ediriomar Peixoto Matos ${ }^{\mathrm{IV}}$
}

DOI: http://dx.doi.org/10.1590/S0102-865020150050000010

IScientific Initiation student, Division of Surgical Technique and Experimental Surgery II, Bahiana School of Medicine and Public Health (EBMSP), Salvador-BA, Brazil. Conception, design, intellectual and scientific content of the study, manuscript preparation.

"Undergraduate student, Medical School, EBMSP, Salvador-BA, Brazil. Acquisition, analysis and interpretation of data; manuscript preparation.

IIIAssociate Professor, Division of Surgical Technique and Experimental Surgery II, EBMSP, Salvador-BA, Brazil. Analysis and interpretation of data, critical revision.

${ }^{\text {IV }} \mathrm{PhD}$, Full Professor, Division of Surgical Technique and Experimental Surgery II, EBMSP, Salvador-BA, Brazil. Conception and design of the study, manuscript writing, critical revision.

\begin{abstract}
PURPOSE: To report the experience of the school in implementing the 3 Rs replace, reduce and refine; showing time and assembling cost of the experimental models used in the teaching of Surgical Technique and Experimental Surgery.

METHODS: Assembly time and costs of models: grafts and flaps performed in pork belly, model of intestinal anastomosis and jejunostomy done in Bahiana box and black box model for training videosurgery.

RESULTS: Average time and cost estimate: ten minutes-pork belly, cost \$ 6.00 per kilogram; two minutes-Bahiana box, cost \$27.2; Black box-3.6 hours for manufacturing, cost $\$ 100.00$. The repetition of each practice the cost is $\$ 3.20$ for Bahiana box and at no cost to the black box.
\end{abstract}

CONCLUSION: The experimental models presented are easily reproducible and of low cost.

Key words: Schools, Medical. Animal Experimentation. General Surgery. Learning Curve. Swine. 


\section{Introduction}

The medical education has been changing in last years. The search for alternative methods at surgical training avoiding animal use, reinforcing ethical principles and also the animal rights. Brazilian education institutions, with ethical concerns, have been adopting means of teaching the practical learning of surgery without compromising quality ${ }^{1}$.

Traditionally, at the Bahiana School of Medicine and Public Health (EBMSP), animal use as biological models was a consolidated practice. However, over the last three years, academic staff from the discipline of Surgical Technique and Experimental Surgery II (TOCE II), decided to develop proper inanimate experimental models, which include training on boxes for open and videosurgery, expanding the quality of education and development of surgical abilities of the academics.

This research has as a purpose to perform an experience report of implementation of the $3 \mathrm{Rs}$, replace, reduce and refine, recommended by Russel and Burch ${ }^{2}$, demonstrate time, cost and assembly processes of the experimental models used on the teaching of operative techniques for medical students at the EBMSP.

\section{Methods}

At the EBMSP, division of Surgical Technique and Experimental Surgery (TOCE II) is divided as theoretical and practical knowledge, the last being the focus of this report. The practical training is performed in a laboratory, which contains nine countertops, where the students group as a trio, which alternate at each experiment between the functions of surgeon, first assistant and surgical instrumentator. Each countertop has a tutor and the professors of the discipline supervise the entire training.

As a method for application of the surgical models on the teaching of operative technique, the practical activities are segmented with three different models: Swine abdomen, used for flaps and grafts practices; Bahiana box, used for intestinal anastomoses and jejunostomy; Black box, experimental model for training in videosurgery ${ }^{3}$ (Figure 1).

The flaps and grafts experiment preparation is made using a $200 \mathrm{~mm} \times 150 \mathrm{~mm}$ swine belly area, fixed on a table with nylon and screws. The incision areas are marked on the skin for the execution of total cutaneous graft, advancement flap, Limberg flap and Z-plasty, as seen in Figure 1A.

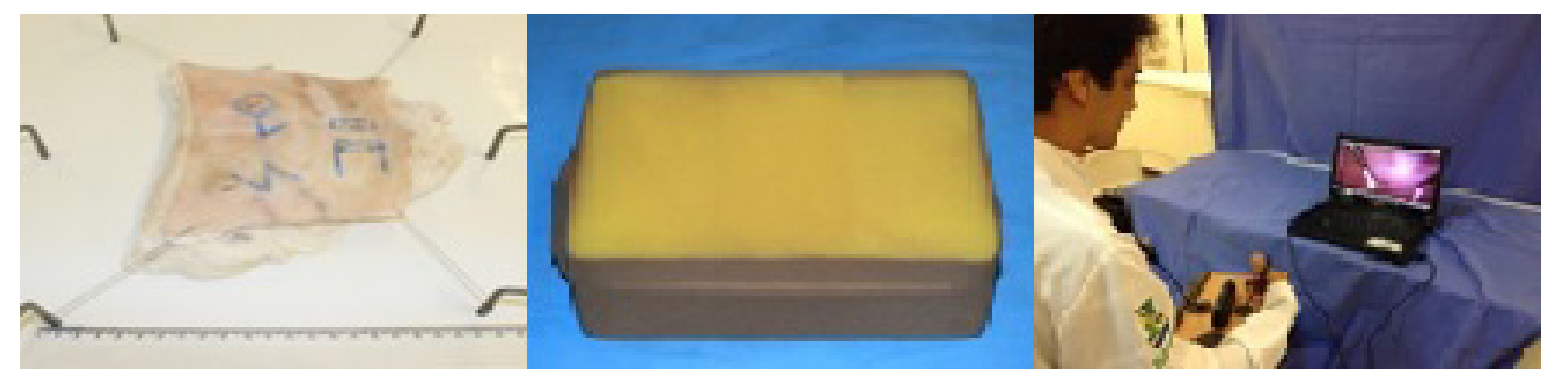

FIGURE 1 - A. Swine abdomen. B. Bahiana box. C. Black box.

At the assembly process of the Bahiana Box, the materials required are: (1) inox box of $200 \mathrm{~mm}$ x $100 \mathrm{~mm}$ x $50 \mathrm{~mm}$, without the cover (Figure 2A); (2) internal coating with cardboard (Figure
2B); (3) three sponges of the brand Limppano ${ }^{\circledR}$ Zapt Dupla Face, with $102 \mathrm{~mm}$ x $69 \mathrm{~mm}$ x $28 \mathrm{~mm}$ each (Figure 2C); (4) half pantyhose (Figure 2D); (5) swine small intestine (Figure 2E). 

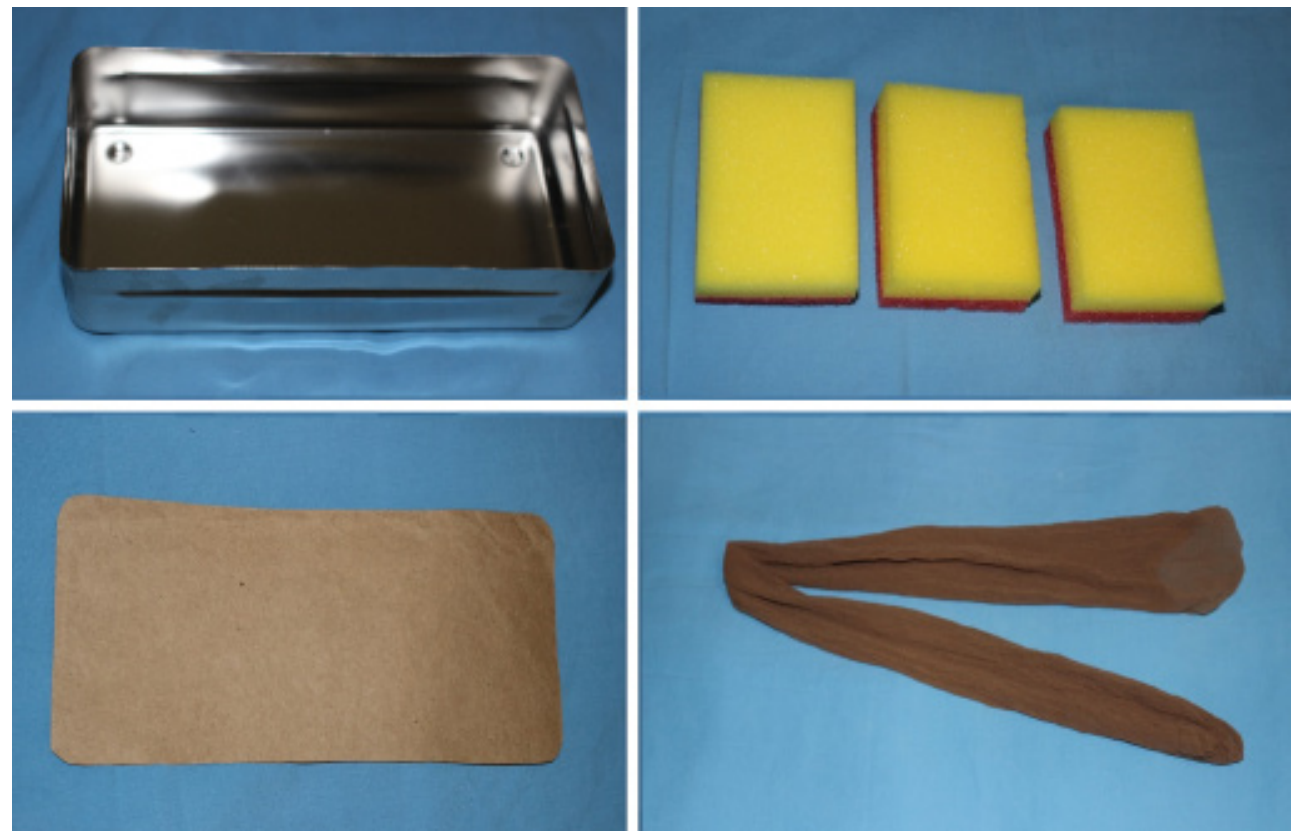

FIGURE 2 - Required materials for surgical model Bahiana box assembly. A. Inox box. B. Cardborad internal coating. C. Three sponges. D. Half pantyhose. E. Swine small intestine.

The assembly process is demonstrated in Figure 3. With this model is possible to create a visceral space for the small intestine and build four layers of incision, represented in Figure 4.

The surgical instruments required for the practical experiments are: scalpel 3 and 4, straight and curved scissors, traumatic and atraumatic tweezers, straight and curved hemostatic tweezers, dynamic retractors, Clamps, Mixters, Allis, compresses, gauzes, Hegar needle holders, absorbable and non-absorbable sutures. These materials are used by demand, thus, are not calculated in this report.
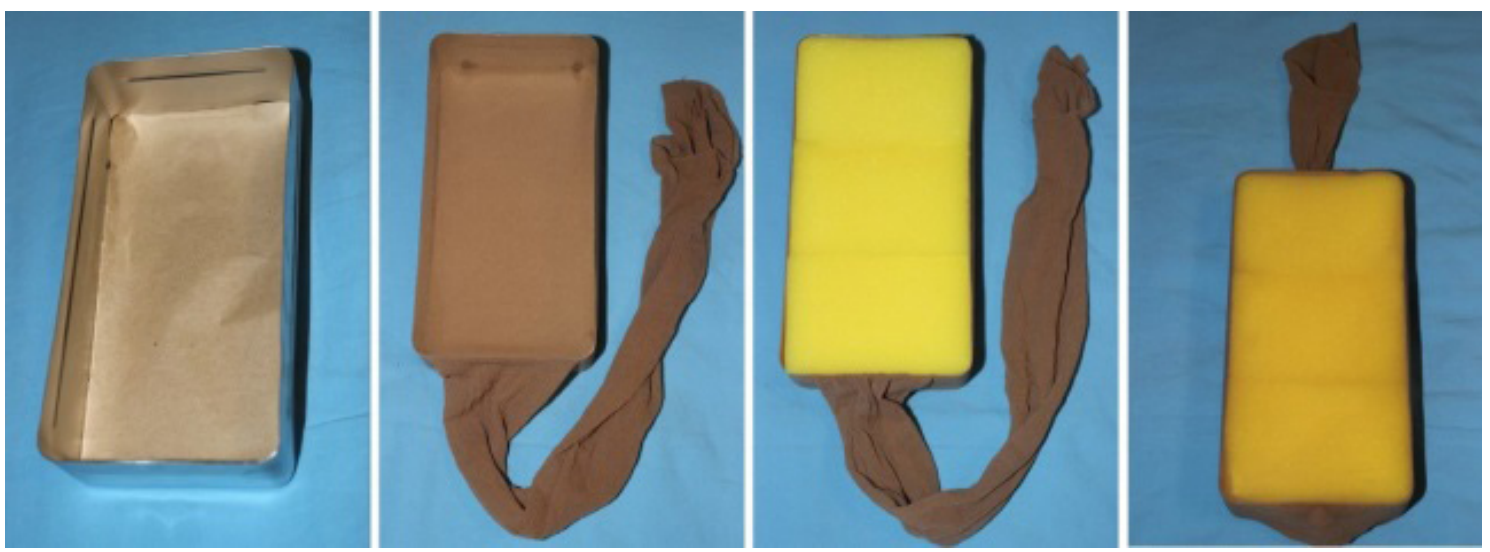

FIGURE 3 - Bahiana box assembly process. A. Cardboard internal coating. B. Pantyhose positioning. C. Sponges positioning. D. Pantyhose superior coating.

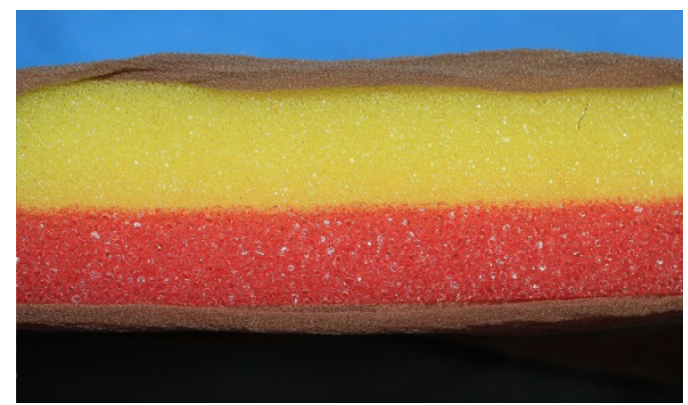

FIGURE 4 - Incision layers. A. Skin (pantyhose). B. Subcutaneous (sponge's yellow side) C. Muscle (sponge's red side). D. Parietal peritoneoum (pantyhose). 


\section{Results}

The estimated mean time of preparation of the experimental model of flaps and grafts was 10 minutes, with the process of fixation in five minutes, and delimitation of the incision areas in more five minutes. The belly swine skin requires a previous preparation, with the cutting of nine sections of skin, $200 \mathrm{~mm} \times 150 \mathrm{~mm}$ each, in a mean time of 30 minutes.

The mean time for assembly of the Bahiana box takes two minutes, as seen on Table 1. The small intestine requires between 10-20 minutes to defrost and be used on the experiments.

TABLE 1 - Bahiana box mean time preparation process.

\begin{tabular}{lc}
\hline Process & Mean Time \\
\hline Internal coating and small intestine positioning & 30 seconds \\
Pantyhose and sponges positioning & 45 seconds \\
Pantyhose superior coating & 45 seconds \\
Total & $\mathbf{1 2 0}$ seconds \\
\hline
\end{tabular}

The black box used by the students on the learning of videosurgery requires 3.6 hours for its assembly, with a meantime of seven minutes to prepare it for the practical experiments ${ }^{3}$. Tables 2 and 3 estimate global costs for obtainment of the models, based on popular market prices, which are of: $\$ 6.00$ per kilogram for the swine belly; $\$ 27.2$ for the Bahiana box (Table 2); $\$ 100.00$ for the black box (Table 3). However, for repetition of each experiment, the Bahiana box needs a $\$ 3.20$ cost, no extra cost with the black box, and the same global cost for the flpas and grafts experiment.

TABLE 2 - Materials and costs required on Bahiana box.

\begin{tabular}{lc}
\hline Material & Cost \\
\hline Inox box of $200 \mathrm{~mm} \times 100 \mathrm{~mm} \times 50 \mathrm{~mm}$ & $\$ 24.00$ \\
Cardboard coating & $\$ 0.04$ \\
Half pantyhose & $\$ 1.80$ \\
Three sponges Limppano® Zapt Dupla Face & $\$ 1.16$ \\
Small intestine & $\$ 0.20$ \\
Total & $\mathbf{\$ 2 7 . 2 0}$ \\
\hline
\end{tabular}

TABLE 3 - Black box estimated budget.

\begin{tabular}{lc}
\hline Material & Cost \\
\hline Wooden box and rubber closures & $\$ 40.00$ \\
Finishing the wooden box & $\$ 20.00$ \\
Video camera & $\$ 20.00$ \\
Support for handling the camera & $\$ 3.00$ \\
Lamp & $\$ 6.00$ \\
Nozzle for lamp & $\$ 3.00$ \\
Eletrical wiring & $\$ 1.00$ \\
Switch & $\$ 2.00$ \\
Male plug for lamp & $\$ 2.00$ \\
Handle for box cover & $\$ 3.00$ \\
Total & $\mathbf{\$ 1 0 0 . 0 0}$ \\
\hline
\end{tabular}

\section{Discussion}

Though there is no universal system to evaluate the surgical abilities precisely, there are three general categories identified for quality evaluation of the surgeon: (1) clinical cognitive abilities, (2) technical abilities, and (3) social interaction ${ }^{4}$. The graduation course must emphasize on methodologies that promote the active participation at the student educational process, early introducing him in practical activities indispensable for the medical career ${ }^{5}$. On this concept, the training provided by the discipline of TOCE II aims to develop and teach surgical principles, abilities and operative techniques similar to those employed at surgical centers and urgency rooms.

The use of swine small intestine and swine belly skin during practical training is shown useful to learn several suture techniques, grafts and flaps, and confection of surgical knots. The limiting factor is the bleeding absence, which restrain the hemostatic training.

Videosurgery is a reality within the medical treatments promoted by diverse specialties, as general surgery, thoracic surgery, neurosurgery and beyond. Thus, it is necessary to stimulate fundamental knowledge and training in this area since the medicine graduation course to not only at the medical residency and at fellowships programs. The black box model developed by EBMSP academics simulates an abdomen in a pneumoperitoneum, allowing training on a bidimensional vision in a monitor without the perception of depth, improving coordination abilities at using the laparoscopic surgical tweezers, and camera control by the auxiliary. The model has a low cost and it can play back the images and movements similar to those performed on videosurgeries ${ }^{3}$. 
Moreover, it is possible to practice basic procedures like object clamping, demountable object manipulation, sutures and knots, appendectomy training, biliary calculus removal, and gastric band.

The described procedures within the models show bioethical advantages, in addition to a less preparation time such as sedation and anesthetic induction are not necessary, less cost due to no vivarium maintenance need, no euthanasia procedures or appropriate carcass discard. Furthermore, the 3 Rs objective is reached avoiding the sacrifice of hundreds of animals and extending the possibilities of academic learning through exhaustive repetition with notorious minimization in the learning curve at the end of the discipline period.

It is noticeable the scarcity of publications that demonstrate the experience and evolution of disciplines of Surgical Technique and Experimental Surgery with the described activity. This study may serve as a stimulation for medical schools to develop new experimental models.

\section{Conclusions}

The experimental models presented are easily reproducible and of low cost. The discipline of TOCE II obtained considerable gains on technical and bioethical scopes at training with experimental inanimate models.

\section{References}

1. Otoch JP, Pereira PRB, Ussami EY, Zanoto A, Vidotti CA, Damy SB. Alternativas ao uso de animais no ensino de técnica cirúrgica. RESBCAL 2012 Jan;1(1):33-40.
2. Balls M, Morton D. FRAME and the Three Rs: Yesterday, Today and Tomorrow. Altern Lab Anim. 2010 Aug;38(4):269-74. PMID: 20822319

3. Batista DM, Felzemburgh VA, Matos EP. New experimental model for training in videosurgery. Acta Cir Bras. 2012 Oct;27(10):741-5. doi: 10.1590/S0102-86502012001000013.

4. Aucar JA, Gronch NR, Troxel SA, Eubanks SW. A review of surgical simulation with attention to validation methodology. Surg Laparosc Endosc Percutan Tech. 2005 Apr;15(2):82-9. doi: 10.1097/01. sle.0000160289.01159.0e.

5. Purim KSM, Santos LDS, Murara GT, Maluf EMCP, Fernandes JW, Skinovsky J. Avaliação de treinamento cirúrgico na graduação de medicina. Rev Col Bras Cir. 2013 Apr;40(2):152-6. doi: 10.1590/ S0100-69912013000200012.

\section{Correspondence:}

Gustavo Maltez de Andrade

Av. Dom João VI, 275

40290-000 Salvador - BA Brasil

Tel.: (55 71)3276-8200

gmaltezdeandrade@gmail.com

Received: Jan 14, 2015

Review: Mar 12, 2015

Accepted: Apr 15, 2015

Conflict of interest: none

Financial source: none

${ }^{1}$ Research performed at Nucleus of Experimental Surgery, Bahiana School of Medicine and Public Health (EBMSP), Salvador-BA, Brazil. 